\title{
Identification and Extraction of Surface Discharge Acoustic Emission Signals Using Wavelet neural network
}

\author{
Nasir A. Al-geelani and M. Afendi M. Piah
}

\begin{abstract}
A hybrid model incorporating wavelet and feed forward back propagation neural network (WFFB-NN) is presented which is used to detect, identify and characterize the acoustic signals due to surface discharge (SD) activity and hence differentiate abnormal operating conditions from the normal ones. The tests were carried out on cleaned and polluted high voltage glass insulators by using surface tracking and erosion test procedure of IEC 60587. A laboratory experiment was conducted by preparing the prototypes of the discharges. This study suggests a feature extraction and classification algorithm for SD classification, which when combined together reduced the dimensionality of the feature space to a manageable dimension. Wavelet signal processing toolbox is used to recover the surface discharge acoustic signals by eliminating the noisy portion and to reduce the dimension of the feature input vector. The test results show that the proposed approach is efficient and reliable. The error during training process was acceptable and very low which attained 0.0074 in only 14 iterations.
\end{abstract}

Index Terms-Acoustic signal, glass insulator, FFB-NN, surface discharge and wavelet transform.

\section{INTRODUCTION}

Atmospheric elements when accumulated on insulator's surface form a layer of pollutant over time. The dielectric properties of the insulator does not diminish significantly, due to the pollutant layer especially when the layer is dry, but due to high humidity, light rain and even fog, it gets wet, and generates a leakage current resulting in a flashover which eventually leads to a disaster in service reliability [1].

The acoustic technology for target detection has developed very rapidly in the past few years. So strong tools are required, such as signal processing and feature extraction for the detection of such a condition [2]. Several researchers successfully used a method of acoustic detection for studying the characteristics of electrical discharges on insulators [3-4]. Many techniques on signal analysis have been used such as Fourier transform, wavelet transform (WT) as well as neural network in order to characterize and classify the electrical discharge signals [5-6]. In some applications, electrical surface discharge detection methods are not very effective, typically as a result of excessive interfering signal [7]. Acoustic method has been used which has advantages over electrical surface discharge detection methods in that they are immune and non-invasive to electromagnetic noise. For the signal analysis, wavelet signal processing was used to

Manuscript received June 15, 2012; revised July 12, 2012.

The authors are with Institute of High Voltage and High Current, Universiti Teknologi Malaysia, 81310 Johor, Malaysia (e-mail: hondahonda750@yahoo.com, fendi@fke.utm.my). de-noise the surface discharge acoustic signal by discarding the noise [8-9]. This paper presents a technique to discriminate between a fault and the other normal operating conditions of the suspension glass insulators by combining wavelet transform with neural network.

\section{The Wavelet Transforms}

The acoustic signals have some non-linear characteristics due to the surface discharge and that makes some difficulties to deal with because of nonlinear and the random like behavior of system. The problem of non-linearity of the acoustic signal is overcome by using wavelet transform which is a strong tool for feature picking-up. It is equivalent to filters. Details (dn) are produce by high pass filters and approximations (an) are produced by low-pass filters. Due to the multidimensional characters which the wavelets possess, there are able to adjust their scale to the nature of the signal features [10]. It can zoom in or zoom out the required details just like a microscope.

Furthermore, wavelets can decompose a signal to give dilations and translations parameters, so the information in the signal is presented by these parameters in the form of frequencies. The matlab wavelet toolbox is used to verify the algorithm where discrete wavelet transform (DWT) are used to analyze the signals. The coefficients are generated and the features from the signal are extracted. Wavelet is a good tool to analyze the non-linear signals as it represents the features both in time and frequency domains [11]. The WT analyses the non-periodic surface discharge signal and adopts the principle of linking of frequency scales. Generally the discrete wavelet transform (DWT) is used for this mission.

\section{BACK-PROPAGATION LEARNING ALGORITHM}

This method was first proposed in 1969 (Bryson and Ho, 1969), but was ignored because of its demanding computations. Only in the mid-1980s was the back-propagation learning algorithm rediscovered. Learning in a multilayer network proceeds the same way as for a perceptron. A training set of input patterns is presented to the network. The network computes its output pattern, and if there is an error - or in other words a difference between actual and desired output patterns - the weights are adjusted to reduce this error. In a perceptron, there is only one weight for each input and only one output. But in the multilayer network, there are many weights, each of which contributes to more than one output [12]. 


\section{EXPERIMENTAL SETUP}

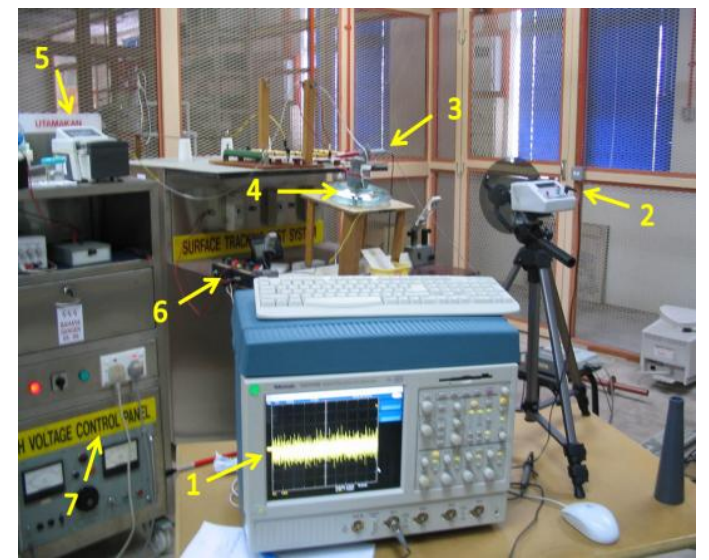

Fig. 1. Experimental setup 1) high resolution digital oscilloscope 2) sensitive ultrasonic detector 3) HV probe 4) HV glass insulator 5) peristaltic pump 6) earth resistor 7) HV AC supply.

Solution of $\mathrm{NaCl}$ was prepared and fed to the peristaltic pump of which the flow rate was adjusted to be very low in order to develop a conducting film on the insulator surface. A high resolution 4-channel digital oscilloscope (Tektronixs TDS55000B series) was used to capture the surface discharge acoustic signals at 5000 samples per second. A sensitive ultrasonic detector with a parabolic antenna was placed on a tripod to capture the acoustic signals. Many trials were done using the experiment setup and the most logical data was finalized

Four test conditions were conducted in the laboratory including first condition in which the insulator was kept clean, second condition in which the insulator was lightly contaminated by a layer of $\mathrm{NaCl}$ solution $(10 \mathrm{mg} / \mathrm{liter})$, third condition in which the insulator was medium contaminated by a layer of $\mathrm{NaCl}$ solution $(20 \mathrm{mg} / \mathrm{liter})$ and fourth condition in which the insulator was heavily contaminated by a layer of $\mathrm{NaCl}$ solution (30mg/liter). In the first condition the insulator was kept clean and the result is shown in Fig. 2. This signal is been processed by using wavelet analysis to remove the noise. It can clearly been seen from the black in color signal that the clean insulator has no or very small surface discharge activity. So this pattern could be considered to be the reference, default pattern or the target to FFB-NN. Fig.3, 4 and 5 shows the second condition, the third condition and the fourth condition and its de-noising signals respectively.

\section{Decomposition of Surface Discharge Signals}

In this work the feature vector is obtained by applying wavelet transform. For a single decomposition the number of coefficients produced by $\mathrm{Db} 2$ is 7 that is 1 approximation coefficient and 6 detailed coefficients. This approximation coefficient that is low frequency component is further decomposed into approximation and detailed coefficients. In using the DWT certain features of the signal that is not immediately obvious in the time domain become more apparent through this multi scale differential operator. The features selected to represent the most important part of the data are: Mean , standard deviation , normalized skewness and normalized kurtosis $\mathrm{k}$, at each decomposition node and were used as a finger-print for each type of SD and as an input to the classifier. The mean , the standard deviation , the normalized skewness and the normalized kurtosis $\mathrm{k}$, were estimated from the following equations:

$$
\begin{gathered}
\eta=\frac{1}{N} \sum_{n=1}^{N}(x[n]) \\
\sigma=\left(\frac{1}{N} \sum_{n=1}^{N}(x[n]-\eta)^{2}\right)^{1 / 2} . \\
\gamma=\frac{1}{N \sigma^{3}} \sum_{n=1}^{N}(x[n]-\eta)^{4} . \\
k=\frac{1}{N \sigma^{4}} \sum_{n=1}^{N}(x(n)-\eta)^{4} .
\end{gathered}
$$

where $\mathrm{x}[\mathrm{n}]$ is the wavelet coefficient at positive $\mathrm{n}$ and $\mathrm{N}$ is the total no. of wavelet coefficients used at each scale. By taking 6-detailed coefficients and 1-approixmation coefficients from the wavelet analysis for the 4-conditions mentioned early, we will have the feature which uses four descriptors for each scale, therefore a feature vector of dimensions was needed to represent the data. So the feature vector is of dimension $42 \times 4$ i.e. 21 samples for the 3-contaminated conditions and other 21 samples for the clean condition that totally gives us 42 dimensional vector each having 4-features. These coefficients are used to train the FFB neural network, so that it should discriminate SD activity from normal operating conditions.

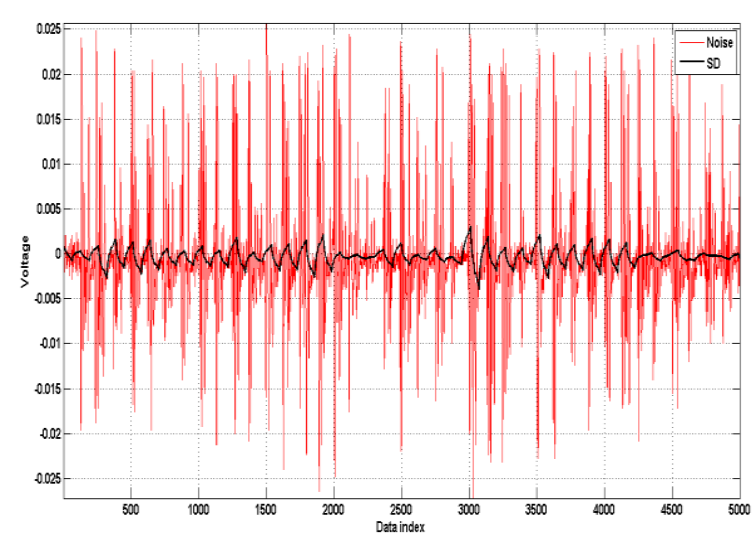

Fig. 2. original and de-noise SD signal of the clean insulator

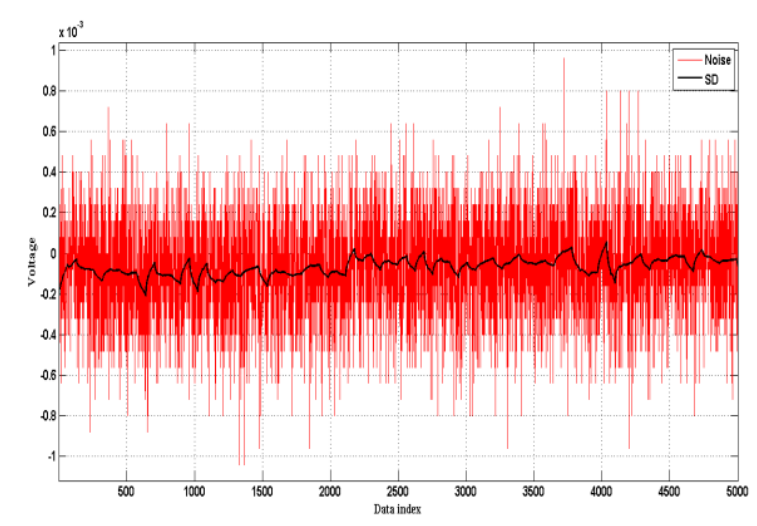

Fig. 3. original and de-noise SD signal of the medium contaminated insulator 


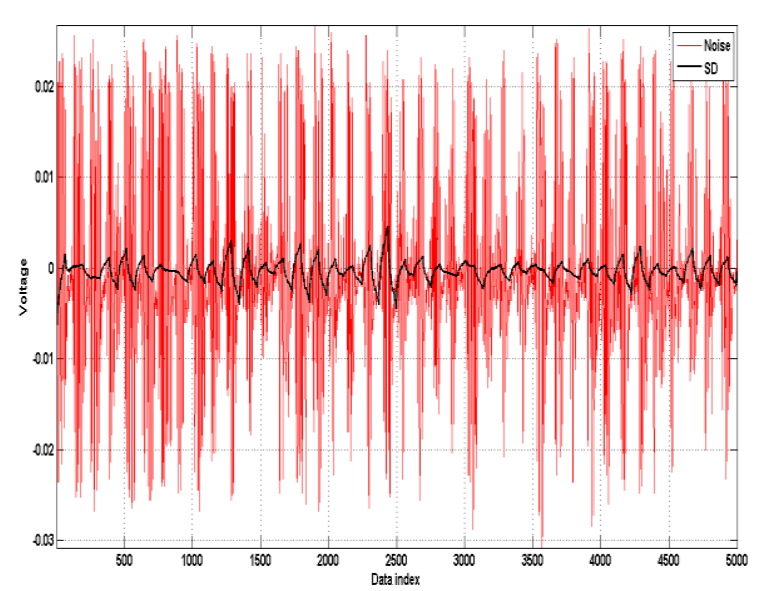

Fig. 4. original and de-noise SD signal of the medium contaminated insulator

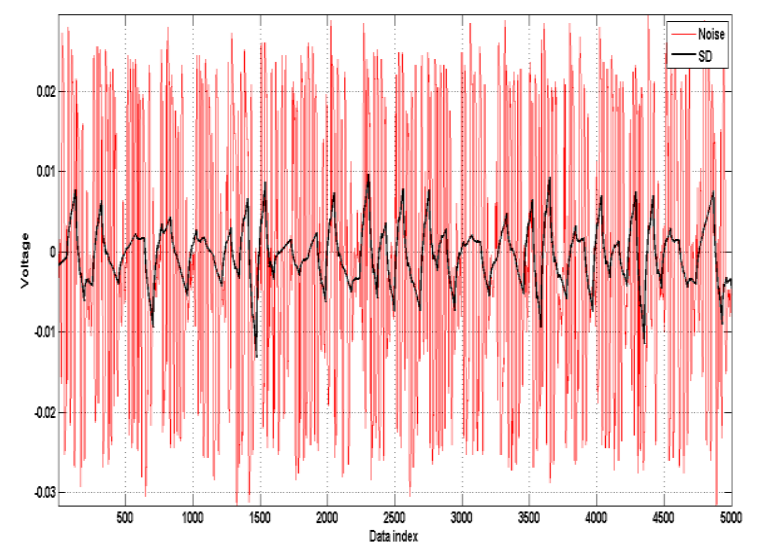

Fig. 5. original and de-noise SD signal of the heavily contaminated insulator

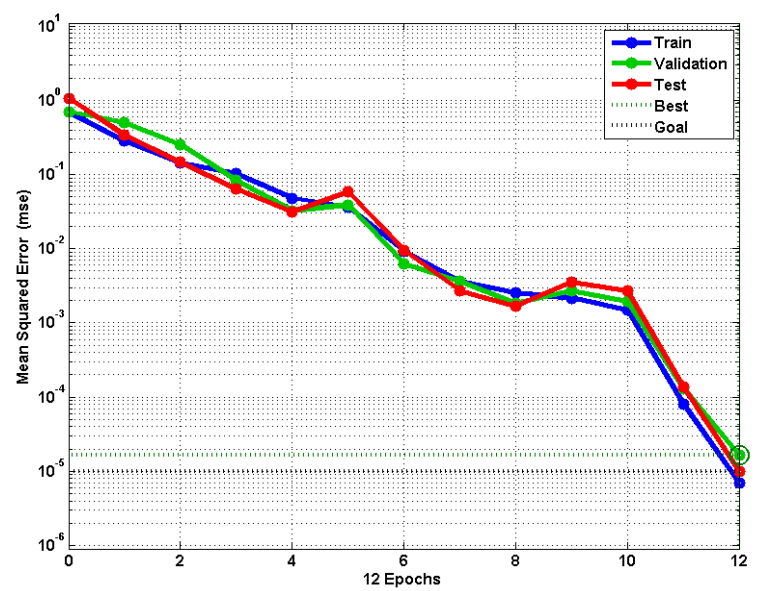

Fig. 6. The training performance

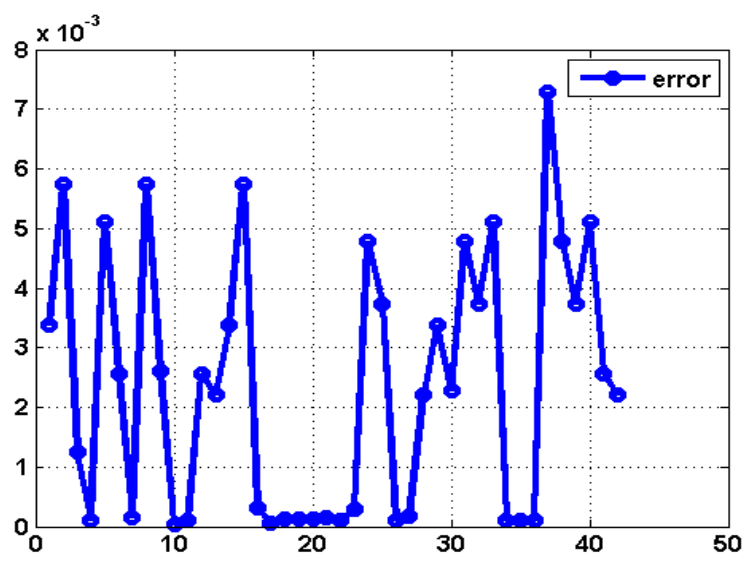

Fig. 7. The training error

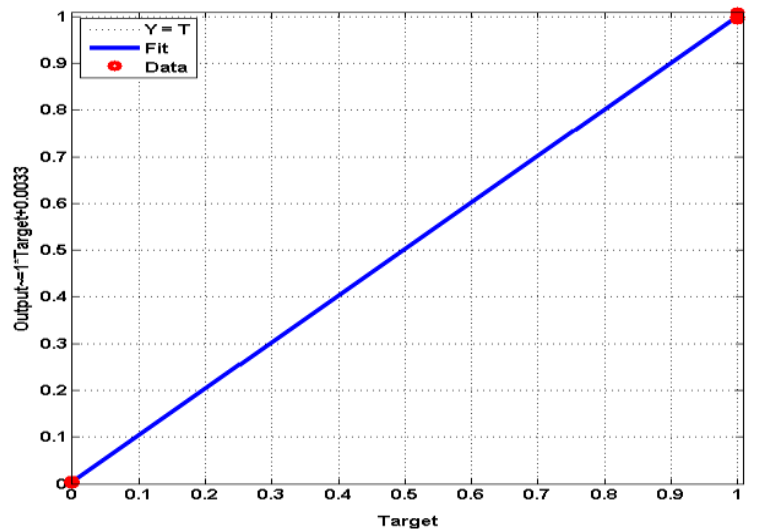

Fig. 8. The regression

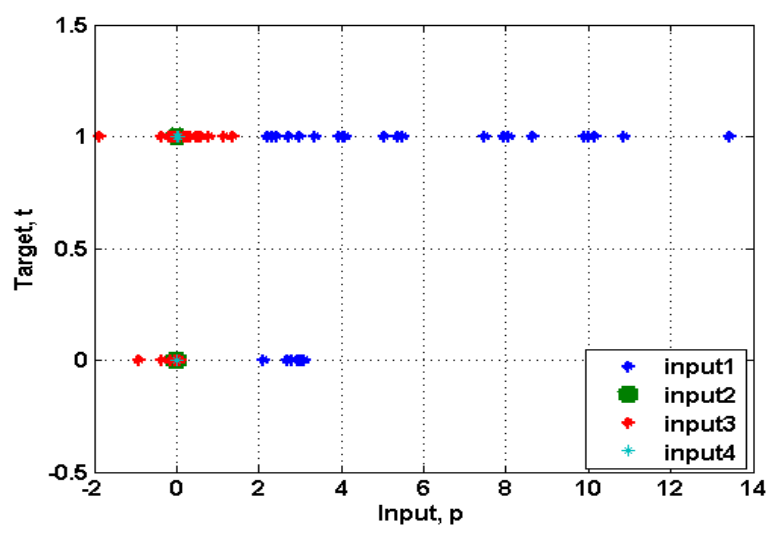

Fig. 9. Testing of FFB-NN by unseen data

\section{RESULTS AND ANALYSIS}

The SD dataset is divided as follows, $(0.6 \%, 0.2 \%, 0.2 \%)$ are selected randomly for training/validation/testing, respectively. By training the FFB-NN with the normalized inputs we can see the result in Fig. 6 which shows us a very perfect training performance. Training performance nearly hit the goal in 12 epochs only giving a very good performance. This means that the neural network had recognized the inputs with a100\% accuracy. The error during training process in Fig. 7 was acceptable and very low which attained 0.0074 in only 14 iterations. The regression in Fig. 8 is approximately 1 that means the fitting is perfect, in which the SD dataset perfectly overlapped the target. The target vector consists of two classes that are designated as: The first class is denoted by (0) which shows no or very small SD activity. The second class is denoted by (1) which shows high SD activity. Testing the FFB-NN is shown in Fig. 9 where the 4-inputs are the features of the SD activity, hence most of the data is accumulated at 1 that means high discharge activity can be found and a small SD activity can be seen at 0 .

\section{CONCLUSION}

The proposed hybrid model has proved to characterize the SD activity with a high degree of integrity which is attributed to the combined effect of the WT and FFB-NN. The high rate of classification acquired by the FFB-NN is due to the preprocessing of data by the WT. The model is quite versatile for a wide range of applications in the field of power system analyses. 


\section{ACKNOWLEDGMENT}

The Authors appreciate the educational and publication support granted from Research Management Center (RMC) Universiti Teknologi Malaysia (UTM), research Vote. No: Q.J130000.7123.01J61 Ministry of Higher Education (MOHE) during the period of this research work.

\section{REFERENCES}

[1] G. Montoya, I. Ramirez, and J. Montoya, "Correlation among ESDD, NSDD and leakage current in distribution insulator," IEE Proceedings Generation Transmission and Distribution, vol. 151, pp. 334-430, 2004.

[2] W. S. Ivan, "A higher density discrete wavelet transform," IEEE Transaction Signal Processing, pp. 3039-3048, 2006.

[3] M. Antonini, M. Barlaud, P. Mathieu, and I. Daubechies, "Image coding using wavelet transforms," IEEE Transaction Image Processing, pp. 205-220, 1992.

[4] R. S. Gorur and J. W. Chang, "Surface hydrophobicity of polymers used for outdoor insulation," IEEE Transactions on Power Delivery, pp. 1923-1933, 1990.

[5] C. Nyamupangedengu, L.P. Luhlanga, and T. Letlape, "Acoustic and HF detection of defects on porcelain pin insulators," in Proceedings of Power Engineering Society, 2007, pp. 1-5.

[6] F. H. Kreuger, E. Gulski, and A. Krivda, "Classification of partial discharges," IEEE Trans-actions on Electrical Insulation, pp. 917-913, 1993.

[7] M. Ugur, D. W. Auckland, B. R. Varlow, and Z.Emin, "Neural network to analyze surface tracking on solid insulators," IEEE Trans. on Electrical Insulation, vol. 4, pp. 763-766, 1997.

[8] T. Zinoulis, A. J. McGrail, D.W. Auckland, B. R. Varlow, I. Argirakis, and W. G. Chadband, "The use of neural networks for discrimination of partial discharges in transformer oil," in Proceedings of Annual Report Conference on Electrical Insulation and Dielectric Phenomena, 1995, pp. 357-360.

[9] Y. Ming and S. Birlasekaran, "Characterization of Partial Discharge Signals Using Wavelet and Statistical Techniques," IEEE Interactional Symposium on Electrical Insulation, 2002.
[10] E. Gulski and F. H. Kreuger, "Computer-aided recognition of discharge sources," IEEE Transactions, 1992, pp. 82-92.

[11] X. Ma, C. Zhou, and I. J. Kemp, "Interpretation of wavelet analysis and its application in partial discharge detection," IEEE Transactions on Dielectrics and Electrical Insulation, pp. 446-457, 2002

[12] M. Negnevitsky, Artificial Intelligence A Guide to Intelligent Systems Second Edition, UK, (2005).

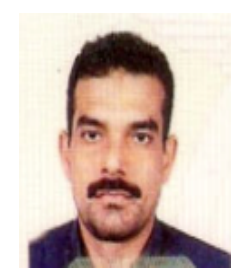

Nasir Ahmed Algeelani holds a masters degree in Electrical Engineering from Universiti Teknologi Malaysia in 2009.

$\mathrm{He}$ has been working as a lecturer at Industrial Technical Institute. (Yemen). At the present he is a research scholar in high voltage engineering at universiti technologi Malaysia. He has recently published a journal paper in Elsevier applied soft computing journal under the title Characterization of acoustic signals due to surface discharges on H.V. glass insulators using wavelet radial basis function neural networks.

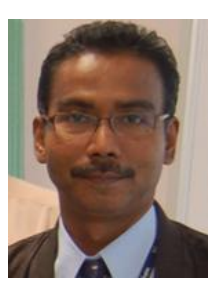

Mohamed Afendi bin Mohamed holds a Ph.D degree in Electrical Engineering Universiti Teknologi Malaysia in 2004. He is a senior lecturer at Faculty of Electrical Engineering, Universiti Teknologi Malaysia and a head of High Voltage Laboratory.

$\mathrm{He}$ is a member of the Institute of Electrical and Electronics Engineers (IEEE), IEEE Dielectrics and Electrical Insulation Society and a collective member of The International Council on Large Electric Systems (CIGRE). He involves in developing a Malaysian Standards documents under the Technical Committee on Electrical Apparatus for Explosive Atmospheres (TCEAEA), SIRIM. His research interests include high voltage insulation diagnostic and co-ordination, partial discharges, polymer insulating materials and insulator condition monitoring. He has published many research papers in journals and conferences. 